\title{
Covertly Probing Underground Economy Marketplaces
}

\author{
Hanno Fallmann, Gilbert Wondracek, \\ Christian Platzer
}


- Significant rise in criminal activity on the Internet

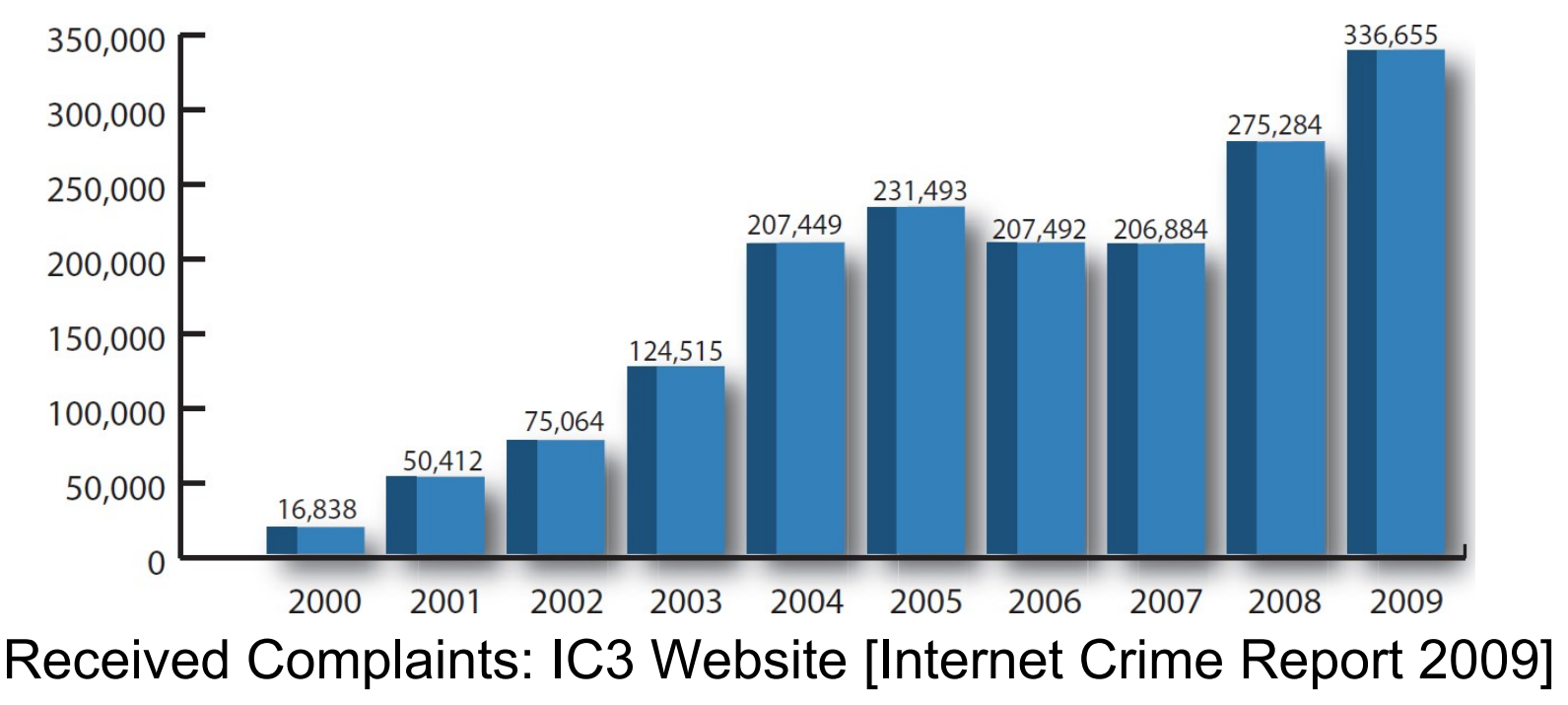

- Cyber-criminals use online communication channels to coordinate themselves and to trade goods and services

- This enables them to specialize in the field of their expertise 
- Researchers and law enforcement have a vital interest in acquiring data about the activities of underground economy marketplaces

- We present a novel system for automatically identifying and covertly monitoring a large number of underground marketplaces simultaneously

- Knowledge about the characteristics of these marketplaces is necessary 


\section{Underground Economy Marketplaces}

- Most interesting marketplaces are

- IRC channels: popular text-based chat protocol

- Web forums: online discussion site

- Both marketplaces are actively policed 


\section{IRC (Internet Relay Chat)}

- IRC networks use customized protocols to add new features, for example:

- Limit on number of messages directed at different targets per time unit

- Enable users to hide their real host address - "vhosting"

- Primitive mechanism to check if user is human

- Primitive check of user-client to thwart bots 


\section{IRC (Internet Relay Chat)}

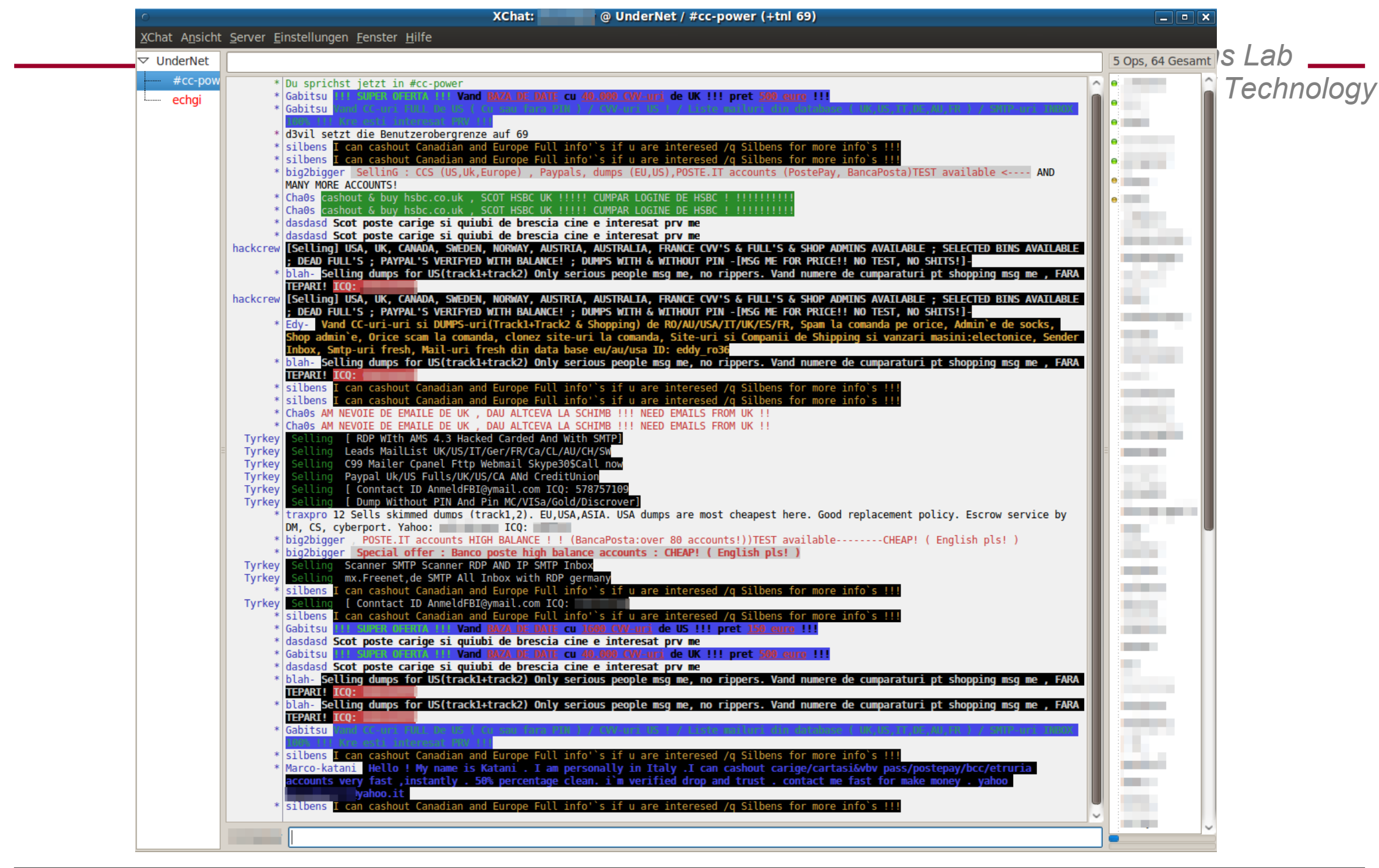

$Q_{0}($ isecLAB) 


\section{Web Forum}

- Interesting content only visible for registered users

- Reputation-based systems and services:

- Trading systems only accessible to members with a minimum reputation rating

- Gain reputation by

- Successfully performing business transactions

- Contribute a certain amount of helpful posts

- Payed a fee

- Escrow services - forum administrators charge a fee to verify the goods 


\section{Credit Card Information}

- Depending on the type of exploit the extend of information that must be known varies:

- Remote exploit: using stolen information to order goods via Internet or telephone. Preferred are "cardable" shops.

- Creating a physical copy: besides having the complete data of a card, a card printer and a hologram is required.

- The price for a card orients itself on the country of origin of the card holder, the type of the card, the volume of the content, and the exclusiveness of the information.

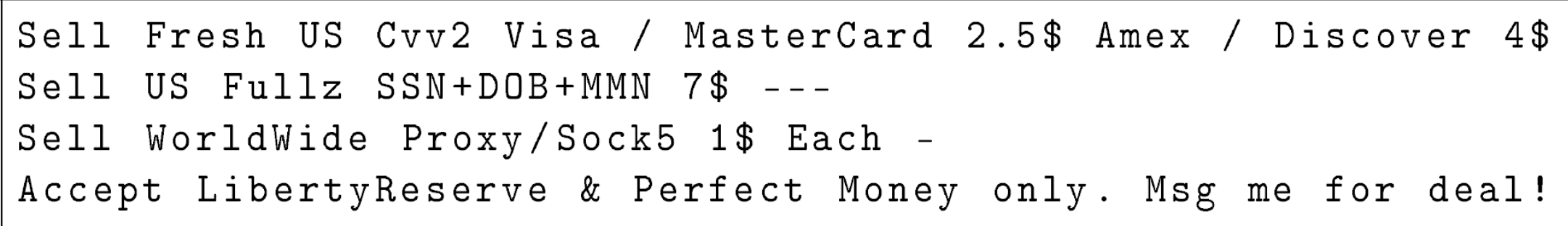




\section{Other Goods and Services}

- Malware

- Malware programs and tool kits are being offered

- Some even include support service

- Boast with anti-detection mechanisms

- Identity Information

- Provide stolen personal information: address, phone numbers, social security number

- Complete Documents: passports, driver licenses, or transportation tickets

- Account Credentials: online games, file hosting services, or social websites 


\section{Underground Economy Advertisements}

I offer serious DDoS attack service from 10 Gbps to 100 Gbps.

I always have between 80,000 and 120,000 bots on my IRC

channel. Type of attack : SYN - TCP - ICMP - UDP - HTTP HTTPS - NEWSYN

I can take down every website even if DDoS protected.

Price start from $200 \$$ USD 24 hours.

AVAILABLE : Free 3 minutes demonstration of attack. 


\section{Sensor System}

Int. Secure Systems Lab Vienna University of Technology

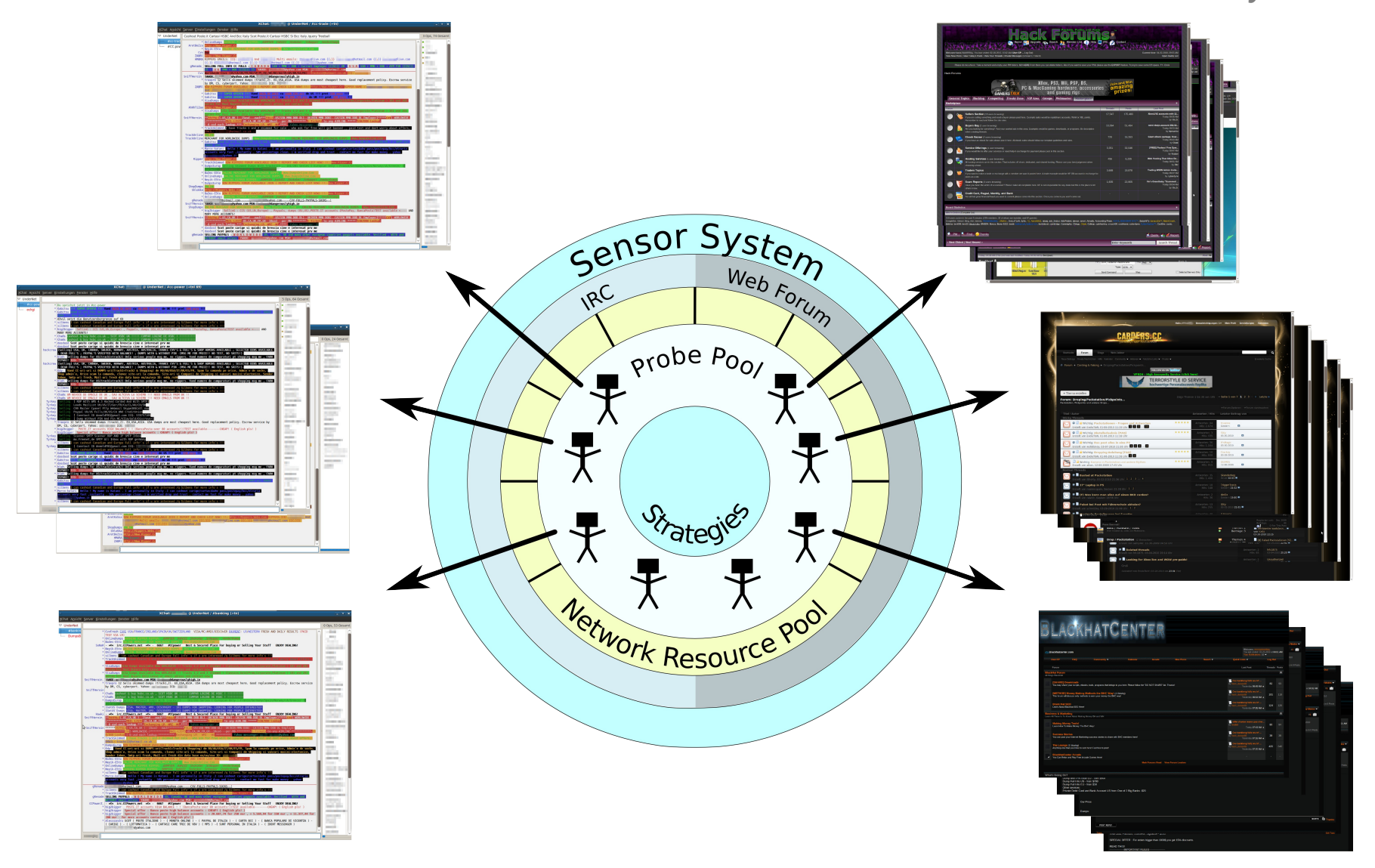




\section{IRC Sensor: Monitoring Strategies}

\section{- Sensor Strategy}

\begin{tabular}{|c|c|c|c|c|c|c|c|c|c|c|c|c|c|}
\hline & $\overbrace{*^{*}}^{*}$ & $\begin{array}{l}*_{1}^{\prime} \\
\text { U } \\
\#\end{array}$ & $\begin{array}{l}{ }^{*} 0 \\
\frac{0}{0} \\
\stackrel{\pi}{*} \\
\frac{*}{*}\end{array}$ & 㞯 & 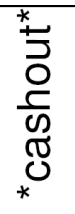 & $\begin{array}{l}\frac{*}{0} \\
\text { 잉 } \\
{ }_{*}^{*}\end{array}$ & 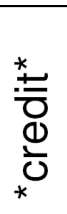 & 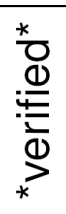 & 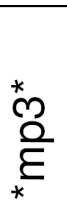 & 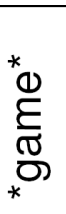 & 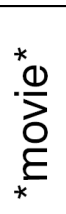 & $\begin{array}{l}{ }^{*} 0 \\
\frac{0}{0} \\
\frac{0}{2}\end{array}$ & \multirow[t]{2}{*}{$\begin{array}{l}\bigotimes \\
\frac{1}{1} \\
\infty \\
0 \\
0\end{array}$} \\
\hline Channel & \multicolumn{8}{|c|}{ Include Patterns } & \multicolumn{4}{|c|}{ Exclude Patterns } & \\
\hline \#cc-trade & & & & & & & & & & & & & $\sqrt{ }$ \\
\hline \#cc-power & & & & & & & & & & & & & $\sqrt{ }$ \\
\hline \#onestopshop & & & & & & & & & & & & & $x$ \\
\hline \#ccvalid & & & & & & & & & & & & & $\sqrt{ }$ \\
\hline \#indocarder & & & & & & & & & & & & & $\sqrt{ }$ \\
\hline \#Mp3traderz & & & & & & & & & & & & & $x$ \\
\hline \#crvcheck & & & & & & & & & & & & & $\sqrt{ }$ \\
\hline \#tradewars & & & & & & & & & & & & & $x$ \\
\hline \#drwho-central & & & & & & & & & & & & & $x$ \\
\hline \#cashout & & & & & & & & & & & & & $\checkmark$ \\
\hline \#carding & & & & & & & & & & & & & $\sqrt{ }$ \\
\hline \#TheCoop & & & & & & & & & & & & & $x$ \\
\hline
\end{tabular}




\section{IRC Sensor: Monitoring Strategies}

- Chain Strategy

- Extend the observation scope by joining unobserved popular channels

- Determine which other channels users of \#banking are currently joined by sending IRC whois requests

- Join the most popular one (\#carding) and apply the same strategy

- Number of newly joined channels limited by configuration

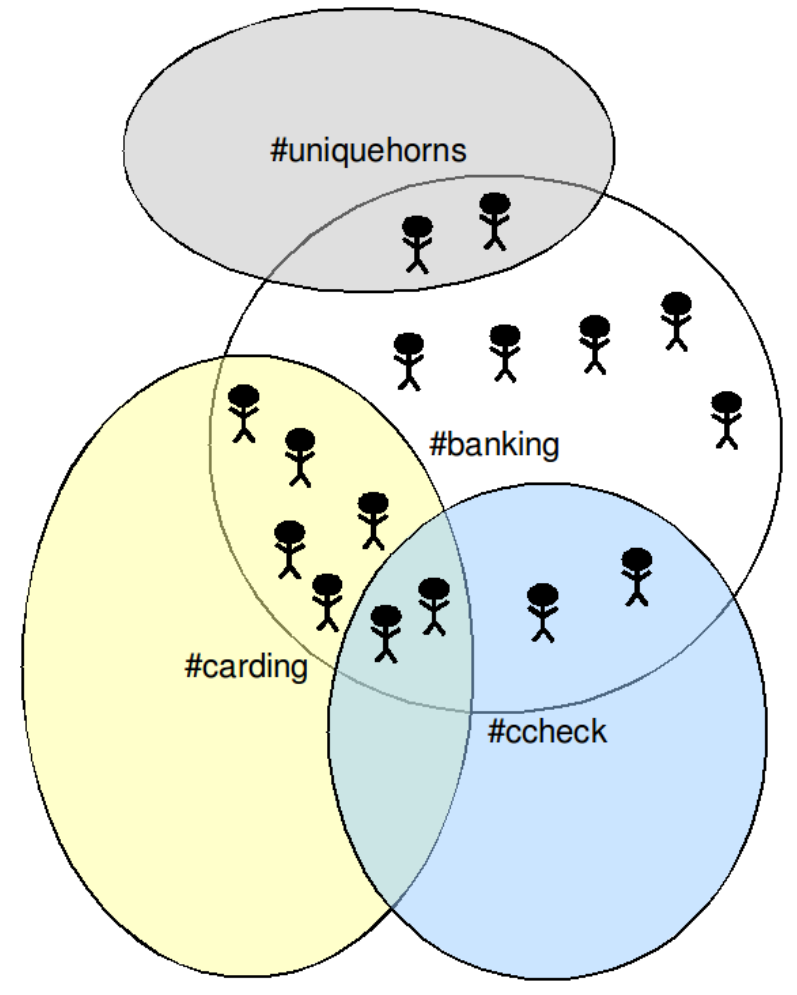




\section{IRC Sensor: Monitoring Strategies}

- Chat strategy

- Cyber-criminals post their advertisements via announcementbots

- Users are asked to request more detailed information

- As soon as we recognize an invitation pattern, we engage the user in a private conversation using an artificial conversational system

- System based on AIML (Artificial Intelligence Markup Language):

Based on patterns, proper responses to incoming message are located 


\section{IRC Sensor Monitoring Strategies}

Channel message:

Trader: \#ccards :i need cvv, to trade it with gud leads, if $u$ have pm me

Private conversation:

Probe: sounds good, need more information too trade Trader: jus need cvv fullz and i trade it with my leads Probe: How badly do you need cvv fullz ? That is interesting. Trader: $i$ need it just to upgrade an account Probe: "cvv fullz"? You need it to upgrade an account? 


\section{IRC Sensor: Monitoring Strategies}

- Swap strategy

- Passive users are frequently removed from IRC channels

- Solution: after a period, we swap the probes with an intentional overlap time

- Combinations

- By combining strategies and applying different observation methods we can construct new strategies 


\section{IRC Sensor: Information Gathering Methods}

- CTCP (Client To Client Protocol)

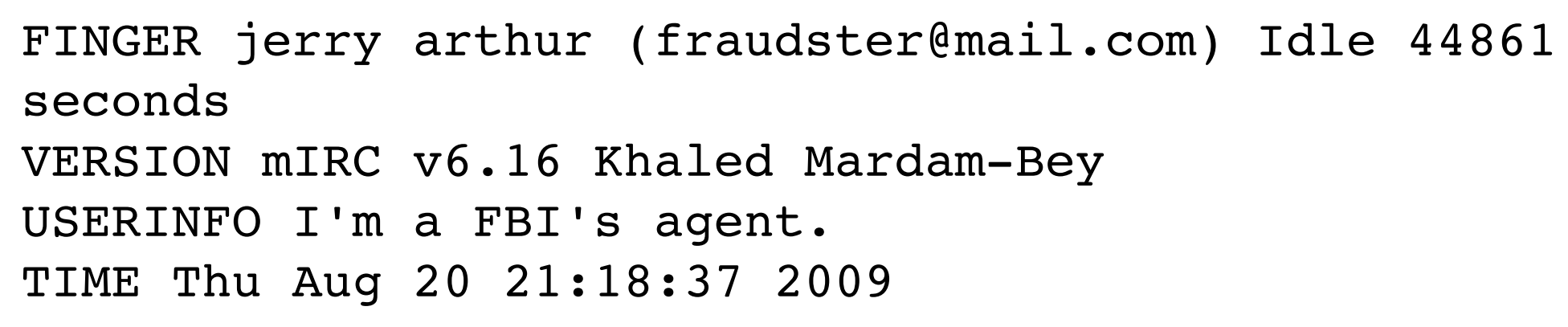

TIME Thu Aug 20 21:18:37 2009 


\section{IRC Sensor: Information Gathering Methods}

- DCC (Direct Client to Client) Protocol

- IRC Whois

311 thedude jeff 192.168.178.1 * : Jeffrey Lebowski

317 xxcarderxx 310171253087663 :seconds idle, signon time

319 Manager : @\#full @\#Ccpower @\#verifications @\#CC2Bank

- IP Address Information

- We can apply tools like Nmap, GeolP, or blacklist lookups 


\section{IRC Sensor: Supervising Information Gathering}

- Some of these strategies are conspicuous $\rightarrow$ have to be applied carefully

- Aim: Avoid causing nuisance or needless traffic

- Solution: Supervisor

- Capable of recognizing affinity of channel to underground economy (Support Vector Machine)

- Automatically dispatches fitting strategies and determines observation methods 


\section{IRC Sensor: Supervising Information Gathering}

- Scanning an IRC network for fraudulent channels:

- Sensor strategy: find obvious trading channels

- Chain strategy: find neighboring popular channels

- Randomly join channels 


\section{Web Forum Sensor}

- Challenges of crawling a web forum:

- Same content has multiple URIs (noisy links) $\rightarrow$ spider trap

- Multitude of forum engines and versions $\rightarrow$ crawler has to be generic but still needs to be capable of recognizing structure

- Solution: crawling techniques described by Yang et al. [Extract Structured Data from Web Forums 2009] 
Threads in Forum : Exchange/Sell/Buy

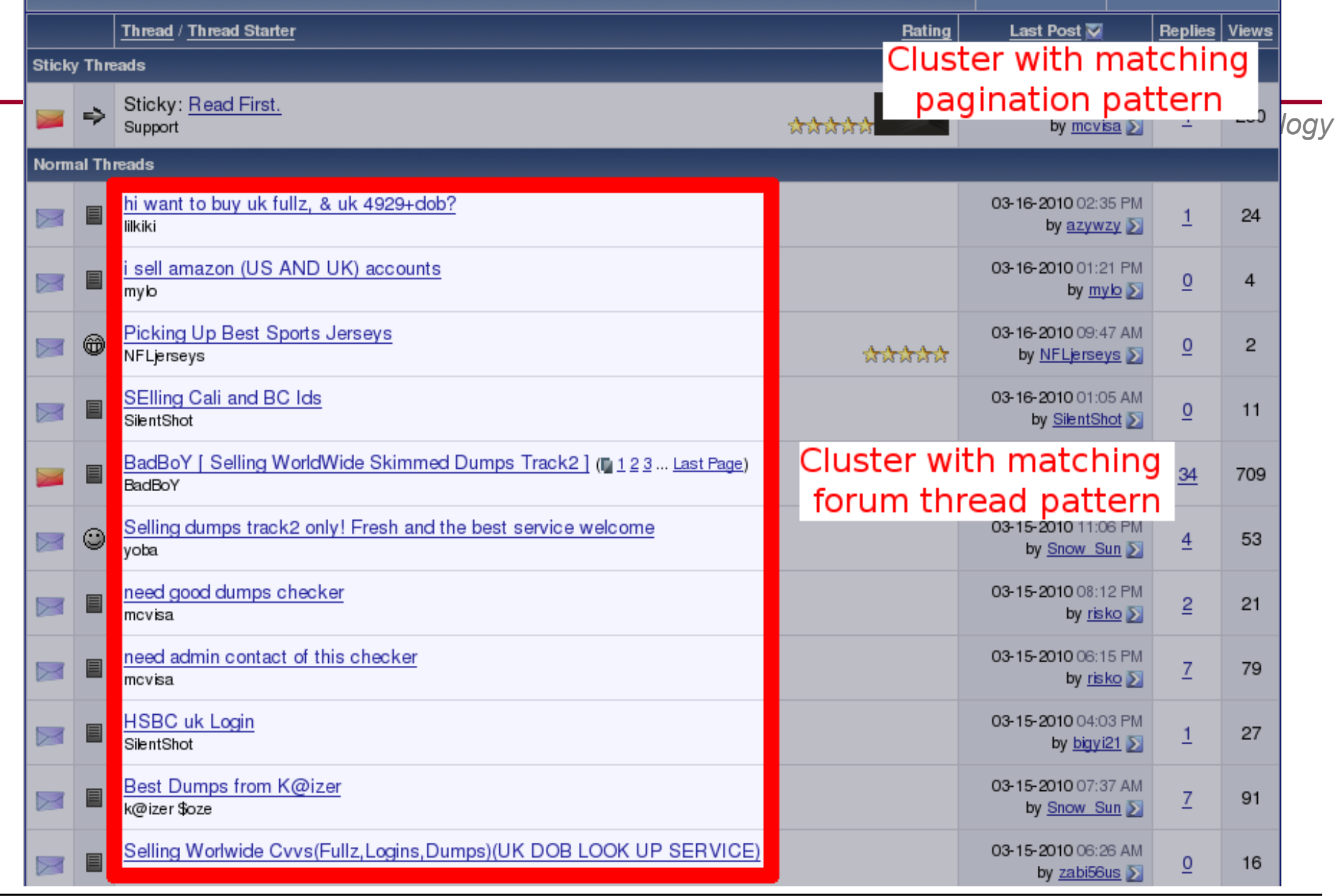




\section{Web Forum Sensor: By-passing Protections}

- Interesting content only available to registered users $\rightarrow$ create them manually

- Limited number of pages per time unit accessible $\rightarrow$ swap user accounts and network addresses

More stealth with distributed crawling 


\section{Evaluation}

- Gathered data for a period of eleven months

- IRC Data:

- We covered 291 IRC networks and found over 495,000 distinct user names

- We observed over 26,000 IRC channels

- 126 channels were recognized by the SVM to be underground economy trading channels

- Chat system started 79 conversations

- 43 million messages related to cyber-crime were recorded $\rightarrow 15$ GB of data 


\section{Evaluation: IRC Scanning Coverage}

$2 \%$ Random

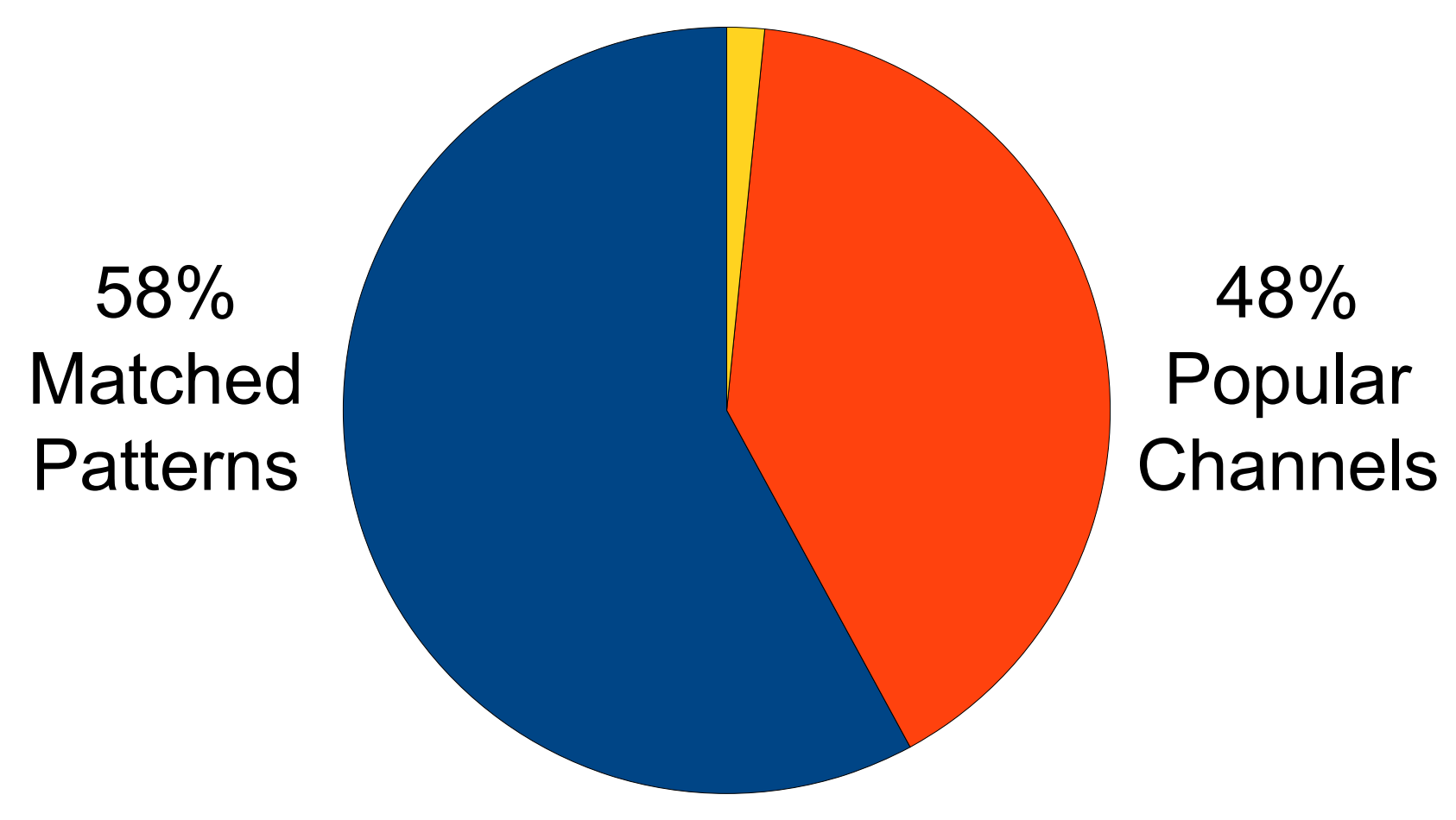




\section{Evaluation: Web Forum}

- Eleven underground forums have been thoroughly analyzed

- Recovered one million posts written by approximately 55,000 users $\rightarrow 127$ GB of data

- Found three types of web forum usages by cybercriminals:

- Spamming legit forums

- Discussion of crime related topics

- Trading illegally obtained goods and offering questionable services 


\section{Questions?}

